\title{
RF MEASUREMENTS RESULTS OF THE FINAL BRAZED SPARC RF DEFLECTOR *
}

\author{
L. Ficcadenti, L. Palumbo, A. Mostacci, University "La Sapienza", Rome, Italy \\ D. Alesini, C. Vaccarezza, G. Di Pirro, LNF-INFN, Frascati, Italy \\ J. Rosenzweig, UCLA, Los Angeles, USA
}

\section{Abstract}

The longitudinal phase space and the horizontal beam slice emittance measurements of the SPARC $150 \mathrm{MeV}-$ $1 n C$ electron beam, foresee the use of a RF deflector. The device is a five cells standing wave structure operating on the TM110-like dipole mode at 2.856Ghz and allows reaching a longitudinal resolution of $\sim 12 u m$ with $2 \mathrm{MW}$ of peak input power. In the paper we illustrate the RF measurements results on the final brazed copper device.

\section{INTRODUCTION}

The characterization of the longitudinal and transverse phase space of the SPARC [1] beam is a powerful tool in order to verify and tune the photoinjector performances. With the use of an RF deflector it is possible to measure the bunch length ([2] [3]) and, adding a dispersive system, the longitudinal beam phase space can be completely reconstructed. In the first section of this paper we briefly review the SPARC RF deflector design parameters. In the second paragraph we report the RF measurement results on the final copper device before the brazing. In the third paragraph we illustrate the adopted tuning procedure and the final RF measurement results on the brazed device. The conclusions are reported in the last section.

\section{RF DEFLECTOR DESIGN PARAMETERS}

A sketch of the complete longitudinal phase space measurement setup of the SPARC beam is shown in Fig. 1. The SPARC bunch is vertically deflected by the RF deflector and horizontally by a magnetic dipole: this allows to completely characterize the energy distribution of each bunch slice reconstructing the longitudinal phase space, as discussed in detail in [4]. The RF Defector is a 5-cell SW

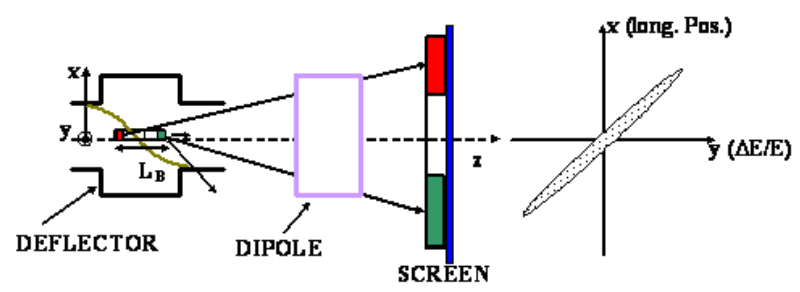

Figure 1: Layout of longitudinal phase space measurement setup.

structure operating on the $\pi$ mode 2 . This structure allows

* This work has been partially supported by the EU in the sixth framework program, Contract no. 011935 EUROFEL-DS1.

06 Instrumentation, Controls, Feedback \& Operational Aspects
Table 1: SPARC RF deflector main dimensions and parameters.

\begin{tabular}{lccc}
\hline Dimensions [mm] & \multicolumn{3}{c}{ Parameters } \\
\hline$b_{2}$ & 60.72 & $f_{\text {res }}[G H z]$ & 2.855961 \\
$b_{1}$ & 59.93 & $Q_{0}$ & 16540 \\
$b_{0}$ & 60.04 & $R_{T}[M \Omega]$ & 2.44 \\
$a$ & 20 & $\frac{R_{T}}{Q}[\Omega]$ & 147 \\
$t$ & 9.5 & $\beta$ & 1 \\
\hline
\end{tabular}

reaching a maximum transverse deflecting voltage of more than $3 M V$ with on input power of $\sim 2 M W$ and a longitudinal resolution lenght of $\sim 12 u m$ [4]. The structure has been design using the electromagnetic codes MAFIA and HFSS. The dimensions and main parameters are reported in Table 1. The radius of the cells has been slightly ad-

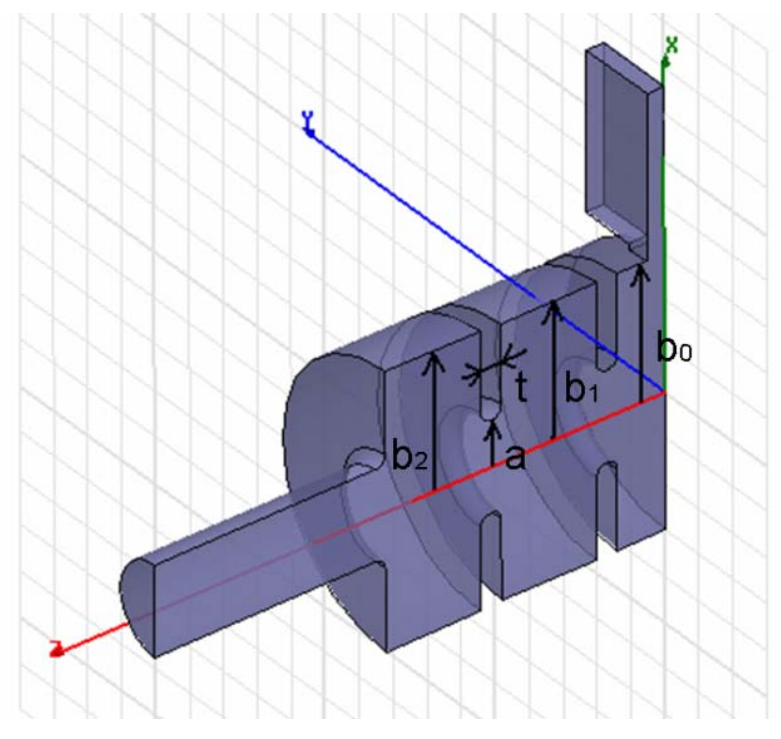

Figure 2: 3D sketch of a quarter of the SPARC RF deflector.

justed in order to obtain a B-field flatness smaller than $5 \%$. Concerning the coupler design it has been inserted in the central cell in order not to exite the nearest mode that have no field in the central cell. The dimension of the coupler window and of the coupler cell radius $b_{0}$ have been tuned in order to obtain a coupling coefficient $\beta=1$ and a resonant frequency equal to $2.856 \mathrm{GHz}$ and to preserve a good $B$ field flatness. The tuning system has been realized using metallic cylinders of $5 \mathrm{~mm}$ radius that can compensate all possible machining errors.

T03 Beam Diagnostics and Instrumentation 1-4244-0917-9/07/\$25.00 (C) 2007 IEEE 


\section{MEASUREMENT RESULTS BEFORE BRAZING}

\section{Resonant frequency and input port coupling mea- surements}

The reflection coefficient at the coupler input port before the tuning procedure is reported in Fig. 3. As expected, it was possible to excite only three of five deflecting modes. The measured external quality factor of the working mode was $Q_{E X T} \cong 17050$, while the measured unloaded quality factor $Q_{0}$ was $\cong 15500$ and was lower than the expected one because of the fact that the structure was simply assembled and not brazed. The corresponding coupling coefficient $\beta$ was $\cong 1$.1.

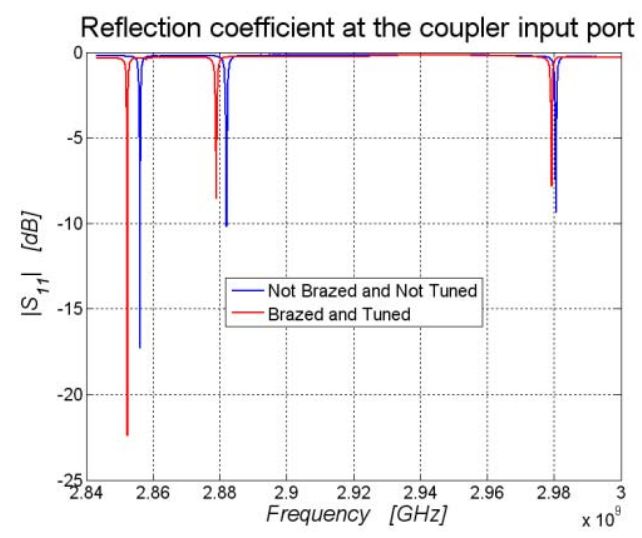

Figure 3: Measured reflection coefficient at the input port.

\section{Field measurement results}

The measurements of the field in the cavity have been done with the bead-pull resonant technique ([5] [6]). The measurement setup with the device before brazing is given in Fig. 4. The PC controls both the network analyzer (NA)

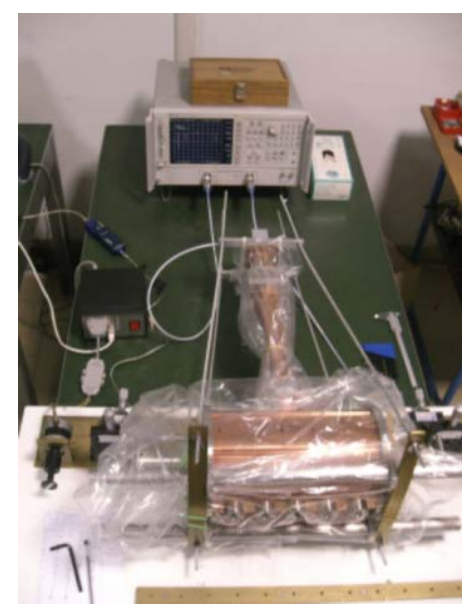

Figure 4: Measurement setup with the device before brazing.

06 Instrumentation, Controls, Feedback \& Operational Aspects
HP8753E and the control circuit of the stepping motor. The nylon wire is kept straight by a $75 g$ weight. Since the the deflection is given by both magnetic and electric fields, both components have to be measured for device characterization. With a small dielectric (teflon) cylinder we have measured the on axis $\mathrm{E}$ field component only while, with a small metallic cylinder both B and E field components. The detailed description of such type of measurements is reported in [4]. We have measured the field flatness of the on axis $\mathrm{H}$ field before brazing the cavity. The deflector were simply assembled by a dedicated metallic cage. The measurement results obtained in air at room temperature of about $22^{\circ} \mathrm{C}$ are reported in Fig. 5, in this case the tuners position were close to the internal surface. The field flatness

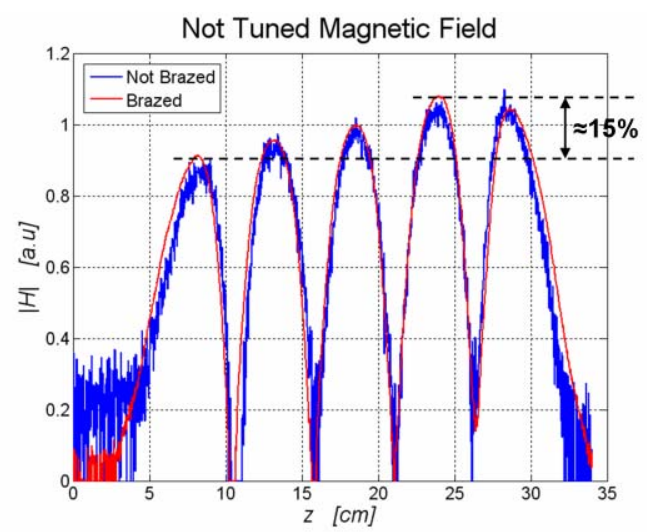

Figure 5: Measured on axis $\mathrm{H}$ field in the not tuned cavity.

was $\cong 15 \%$ and the resonant frequency $\cong 2.8563 \mathrm{GHz}$ in good agreement with expectations even if a dedicated tuning was necessary to obtain a field flatness of the order of $5 \%$.

\section{MEASUREMENT RESULTS AFTER BRAZING AND FINAL TUNING}

After the cavity brazing, the device has been installed in SPARC (Fig. 6) to tune the structure at the working temperature of $45^{\circ} \mathrm{C}$. The measurements of the $\mathrm{H}$ field profile is

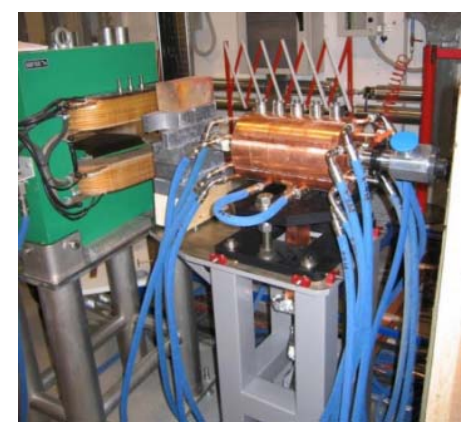

Figure 6: RF deflector installed in SPARC.

reported in Fig 5. As the plots shows, the brazing procedure T03 Beam Diagnostics and Instrumentation 
has not modified the structure dimensions. The measurements before brazing is more noisy because we used a reduced NA input power. The $\mathrm{H}$ field measurements and the tuning has been done in air. For this reason, we tuned the structure at the frequency of $f_{\text {res }}^{\text {air }} \cong 2.8551 \mathrm{GHz}$. To tune the deflector we have linearized the field profile sensitivity and the resonant frequency as a function of the tuner positions around the initial working point following the steps described below:

- we have measured $\mathrm{H}$ field peaks of each cell $\left(H_{0, i}\right)$ at the initial resonant frequency $\left(f_{\text {res } 0}\right)$;

- we have measured the sensitivity coefficients of the $\mathrm{H}$ field $\left(\frac{\partial H_{i}}{\partial t_{j}}\right)$ and of the resonant frequency $\left(\frac{\partial f_{r e s}}{\partial t_{j}}\right)$ as a function of tuner positions;

- to find the required relative tuner variations $\left(\Delta t_{j}\right)$ we have resolved the following system:

$$
\left\{\begin{array}{l}
H_{0, i+1}-H_{0, i}=\sum_{j=1}^{5}\left(\frac{\partial H_{i}}{\partial t_{j}}-\frac{\partial H_{i+1}}{\partial t_{j}}\right) \cdot \Delta t_{j} \\
f_{\text {res }}-f_{\text {res } 0}=\sum_{j=1}^{5}\left(\frac{\partial f_{r e s}}{\partial t_{j}}\right) \cdot \Delta t_{j}
\end{array}\right.
$$

where $i=1, \cdots, 4$. and $f_{\text {res }}=2.8551 G H z$ As example, the resonant frequency as a function of the first tuner position is reported in Fig. 7. In the same plot it is reported the linearized curve that gives the frequency sensitivity. Fig. 8

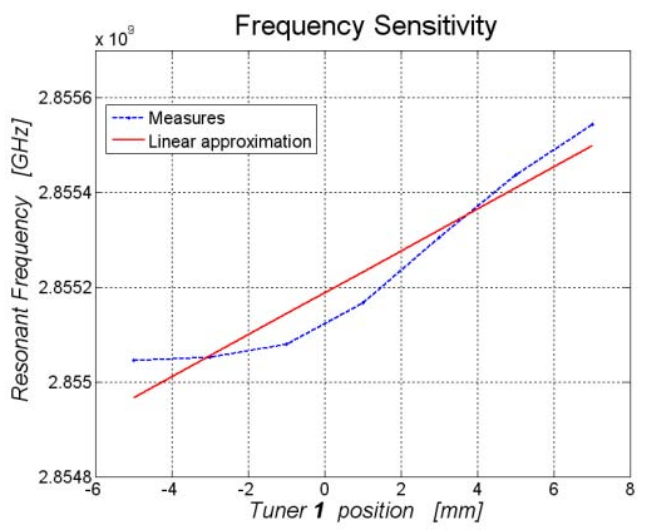

Figure 7: Frequency sensitivity at different tuner positions.

shows the $\mathrm{H}$ field amplitude in the first cell as a function of the tuner positions. The final tuned field profile at the frequency of $2.8551 \mathrm{GHz}$ is reported in Fig. 9. The reached field flatness is $\cong 4 \%$, according with the SPARC RF deflector requirements [4]. The final reflection coefficient at the coupler input port after brazing and tuning is reported in Fig. 3. The corresponding unloaded quality factor and coupling coefficient are $\cong 16000$ and $\cong 1.1$ respectively.

06 Instrumentation, Controls, Feedback \& Operational Aspects

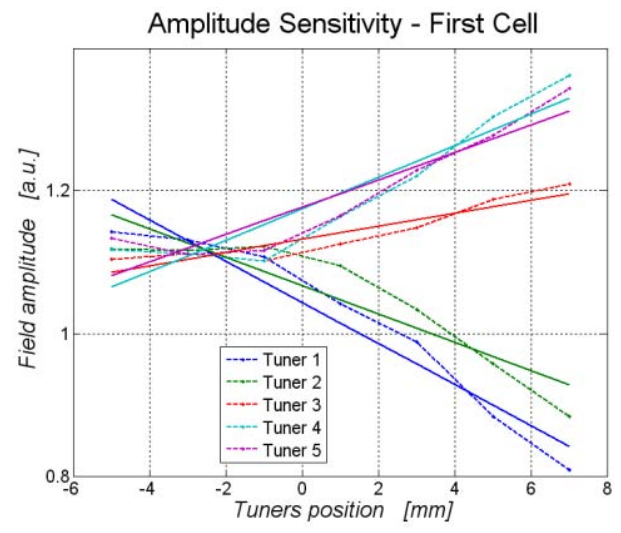

Figure 8: First cell field amplitude sensitivity at different tuner positions.

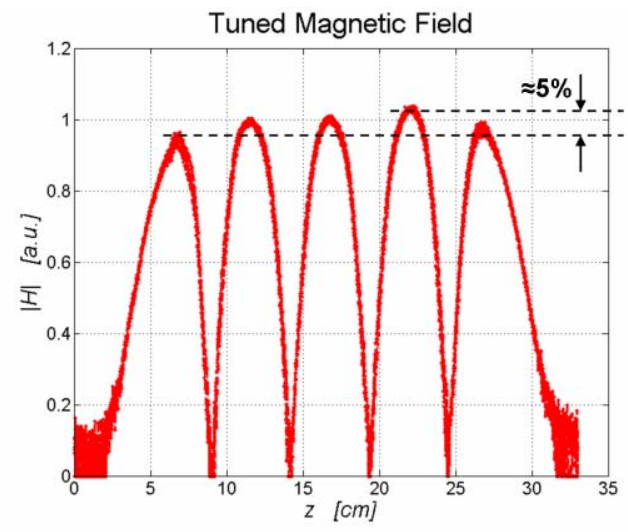

Figure 9: Measured on axis $\mathrm{H}$ field in the brazed tuned cavity.

\section{CONCLUSIONS}

The SPARC RF deflector as been successfully designed, realized and brazed at LNF-INFN. It is a five cell SW structure operating at $2.856 \mathrm{GHz}$ made in copper. A complete RF characterization using bead-pull and scattering coefficient measurements, has been done before and after brazing. A dedicated tuning procedure has been implemented to reach a field flatness of few percent at the designed resonant frequency.

\section{ACKNOLEDGEMENTS}

The authors would like to thank A. Clozza, V. Lollo and R. Di Raddo for mechanical drawings, realization and technical support.

\section{REFERENCES}

[1] Preliminary TDR of SPARC, www.lnfn.infn.it, 2004.

[2] G.A. Loew, O.H. Altenmuller, SLAC, PUB-135, August 1965.

[3] P. Emma, et. al. LCLS-TN-00-12, 2000.

[4] D. Alesini, et. al.,NIM, A 568 (2006) 488-502.

[5] L.C. Maier,J.C. Slater, J. Appl. Phys.,23 (1) (1951).

[6] F. Caspers,G. Dome, CERN SPS/85-46 (ARF), 1985.

T03 Beam Diagnostics and Instrumentation 